MITSUBISHI ELECTRIC RESEARCH LABORATORIES

http://www.merl.com

\title{
Coded Modulation Design for Finite-Iteration Decoding and High-Dimensional Modulation
}

\author{
Koike-Akino, T.; Millar, D.S.; Kojima, K.; Parsons, K \\ TR2015-016 March 2015
}

\begin{abstract}
We show how to design capacity-approaching LDPC codes under different modulation formats and different decoding methods. With EXIT chart analysis for finite-iteration BICM-ID of various HDM, we achieve $2 \mathrm{~dB}$ gain from a code optimized for 1D modulation.
\end{abstract}

Optical Fiber Communication Conference and Exposition (OFC)

This work may not be copied or reproduced in whole or in part for any commercial purpose. Permission to copy in whole or in part without payment of fee is granted for nonprofit educational and research purposes provided that all such whole or partial copies include the following: a notice that such copying is by permission of Mitsubishi Electric Research Laboratories, Inc.; an acknowledgment of the authors and individual contributions to the work; and all applicable portions of the copyright notice. Copying, reproduction, or republishing for any other purpose shall require a license with payment of fee to Mitsubishi Electric Research Laboratories, Inc. All rights reserved. 



\title{
Coded Modulation Design for Finite-Iteration Decoding and High-Dimensional Modulation
}

\author{
Toshiaki Koike-Akino, David S. Millar, Keisuke Kojima, Kieran Parsons \\ Mitsubishi Electric Research Labs. (MERL), 201 Broadway, Cambridge, MA 02139, USA. \\ koike@merl.com
}

\begin{abstract}
We show how to design capacity-approaching LDPC codes under different modulation formats and different decoding methods. With EXIT chart analysis for finite-iteration BICM-ID of various HDM, we achieve 2dB gain from a code optimized for 1D modulation.
\end{abstract}

OCIS codes: (060.4510) Optical communications, (060.1660) Coherent communications, (060.4080) Modulation.

\section{Introduction}

Recent advancement of forward error correction (FEC) codes based on low-density parity-check (LDPC) codes [1-6] has convinced us that capacity-achieving performance is no longer idealistic. However, a well-designed LDPC code at a certain condition cannot always perform well for different conditions. Ignoring this reality, one may often use one best-known LDPC code for different high-order modulations, different high-dimensional modulations, different iteration decoders, with and without iterative demodulations (ID), or different fiber plants. In this paper, we emphasize that a significant benefit up to $2 \mathrm{~dB}$ can be achieved by re-designing LDPC codes for different conditions. In particular, we consider two cases; one is an iteration-dependent design of bit-interleaved coded modulation (BICM) for different number of iterations in belief-propagation (BP) decoding, and the other is a modulation-dependent design of BICM iterative demodulation (BICM-ID) with different high-dimensional modulations (HDM) [7-12].

\section{Iteration-Dependent LDPC Code Design for BICM with Finite-Iteration BP Decoding}

Good LDPC codes can be typically obtained by designing degree distributions with density evolution (DE) or extrinsic information transfer (EXIT) chart [4]. For example, degree optimization is performed by linear programming for EXIT curve fitting. This methodology works well when the number of BP iterations is very large. The larger number of iterations can lead to better performance in general. However, practical decoders often cannot iterate many times in optical communications because data throughput and power consumption have been extremely high [13]. We first focus on degree optimization via EXIT chart for a finite-iteration BP decoder. Our result suggests that an LDPC code for BICM should be different when the BP decoder changes the maximum number of iterations to control throughput and power consumption. If we do not follow this rule, we may suffer from up-to $1.8 \mathrm{~dB}$ penalty.

Instead of fitting EXIT curves, we can optimize degree distributions by tracking mutual information updates trajectory at each iteration count of EXIT chart to minimize the required signal-to-noise ratio (SNR), a.k.a. threshold. The results of this iteration-aware LDPC code design are shown in Fig. 1(a), in which we plot the threshold as a function of the maximum number of BP iterations for check-concentrated triple-weight irregular LDPC codes with a code rate of 0.8. The check-concentrated triple-weight LDPC codes [3] were shown to achieve very good performance as mentioned in [4]. Although it is known in [2] that larger maximum degree of variable nodes offers better threshold, the decoder complexity becomes higher. Therefore, we consider the maximum degree of variable nodes below 16 so that high-throughput decoder can be implemented. The LDPC code designed by conventional curve fitting provides the best performance if the decoder can iterate more than 100 times, while the threshold seriously degrades for the cases of fewer iterations. For such fewer iteration decoders, we shall use different irregular LDPC codes. For example, the LDPC code optimized for 8-iteration decoder outperforms the conventionally optimized LDPC code by $1.1 \mathrm{~dB}$, and the LDPC code optimized for 4-iteration decoder offers $1.8 \mathrm{~dB}$ better threshold. On the other hand, the 4-iteration optimized LDPC code has approximately $0.8 \mathrm{~dB}$ loss from the conventionally optimized LDPC code when the decoder can iterate more than 100 times.

Using progressive edge-growth (PEG) [14], we designed finite-length high-girth parity-check matrix according to the optimized degree distributions. The bit-error rate (BER) performance of those iteration-dependent LDPC codes (whose codelength is 38400) is shown in Fig. 1(b) for finite-iteration BP decoders. Here, we consider dual-polarization 


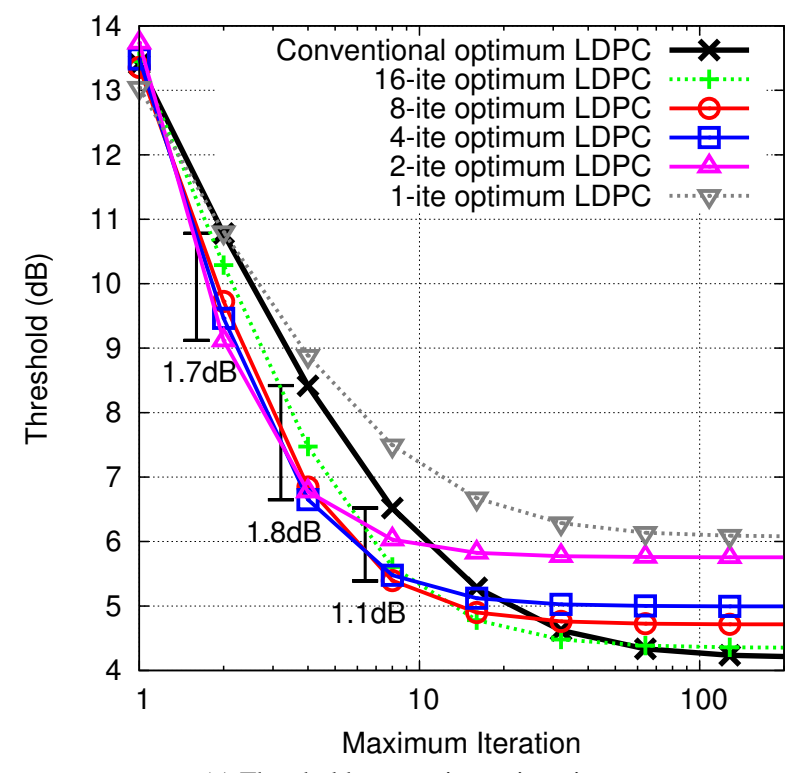

(a) Threshold vs. maximum iteration

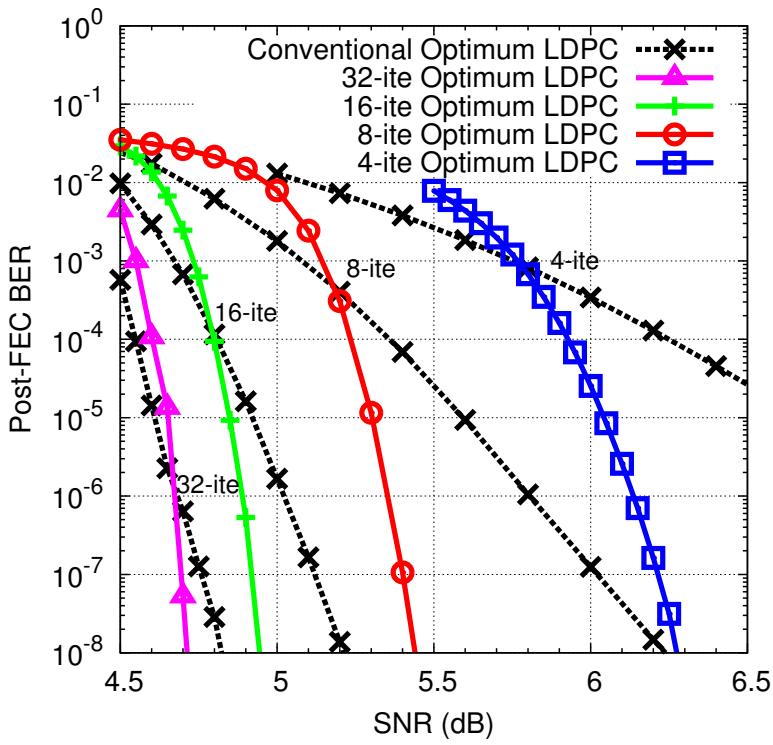

(b) BER vs. SNR

Fig. 1: Iteration-dependent LDPC design for BICM with finite-iteration BP decoding.

quadrature phase-shift keying (DP-QPSK) in back-to-back simulations. As expected in the above-mentioned threshold analysis, the conventionally optimized LDPC code does not perform well for fewer iteration BP decoding, in which the BER slope becomes worse. Our iteration-aware LDPC codes perform much better for each cases. For 8-iteration BP decoder, the required SNR at a BER of $10^{-8}$ of the conventionally optimized code has a loss of $0.8 \mathrm{~dB}$ compared to our 8-iteration optimized LDPC code. This gap must be much more significant at a BER below $10^{-15}$.

\section{Modulation-Dependent LDPC Code Design for BICM-ID with High-Dimensional Modulation}

We now consider the second example of modulation-dependent LDPC code design for HDM. HDM has received a lot of interest in the community because of its high sensitivity [7-12]. However, our EXIT chart analysis of lattice packing in [11] showed that higher dimensionality cannot always provide higher mutual information, while higher improvement is expected with a priori information. This implies that we may need BICM-ID to fully exploit the increased dimensionality. It is because such a lattice-packed HDM just maximizes the minimum Euclidean distance and labeling problem is hard to solve for BICM. We again exemplify that one LDPC code optimized for 1D modulation is no longer optimal for HDM with BICM-ID.

With EXIT chart analysis of various HDM based on sphere-cutting lattice [10,11], we optimized degree distributions of LDPC codes. The threshold analysis of those optimized degree distributions is shown in Fig. 2(a), where threshold penalty from Shannon limit is presented as a function of dimensionality for two cases of BICM and BICM-ID. Here, we again considered check-concentrated triple-weight LDPC codes, whose maximum degrees of variable nodes are below 16. For BICM-ID, we used a methodology proposed in [4], in which EXIT curves of variable-node decoder is combined with that of demodulator. As shown in this figure, higher dimensionality may improve the threshold for the case of 1 bit-per-symbol HDM with a code rate of 8/9. Note that the threshold gap between BICM-ID and BICM for 24 dimensions (24D) is approximately $1 \mathrm{~dB}$. More importantly, when we use higher-order HDM, the threshold degrades with the increased dimensionality for BICM (not BICM-ID). Although higher order modulations even without HDM approach Shannon limit if lower code rates are available, the decoder complexity per information bit can be larger for low-rate LDPC codes.

Sometimes, one LDPC code optimized for 1D modulation is also used for HDM either with BICM or BICM-ID. However, if we do not use different LDPC codes depending on BICM or BICM-ID, we may need to pay some penalties. We show post-FEC BER performance of 24D block-coded modulation [10] in Fig. 2(b), where we use 32 iterations in BP decoding for two LDPC codes (designed by PEG for a codelength of 38400 and a code rate of 8/9); one is the degree distribution [3] designed for BICM and the other is our optimized degree distribution designed for BICM-ID 


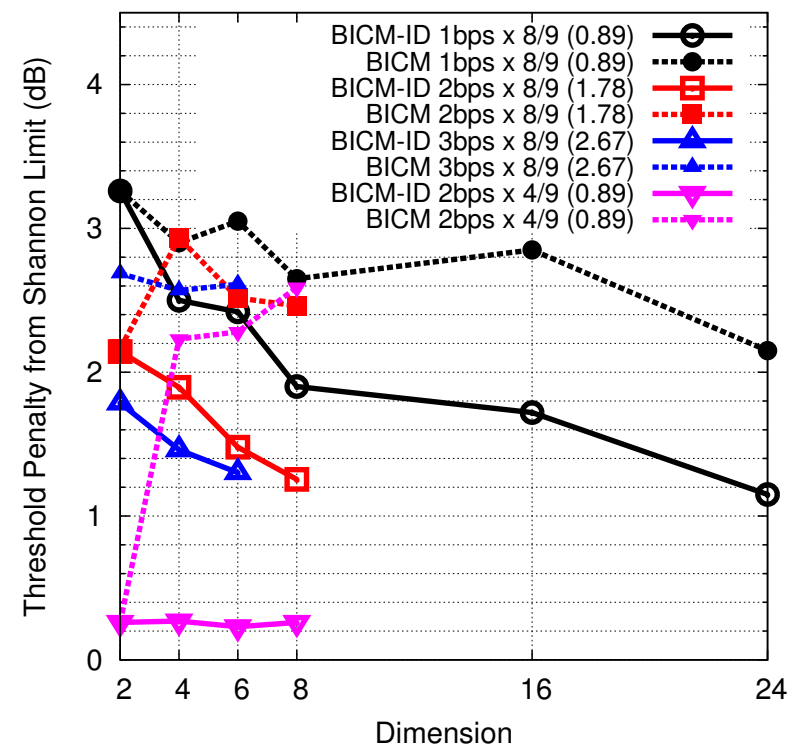

(a) Threshold vs. dimensionality

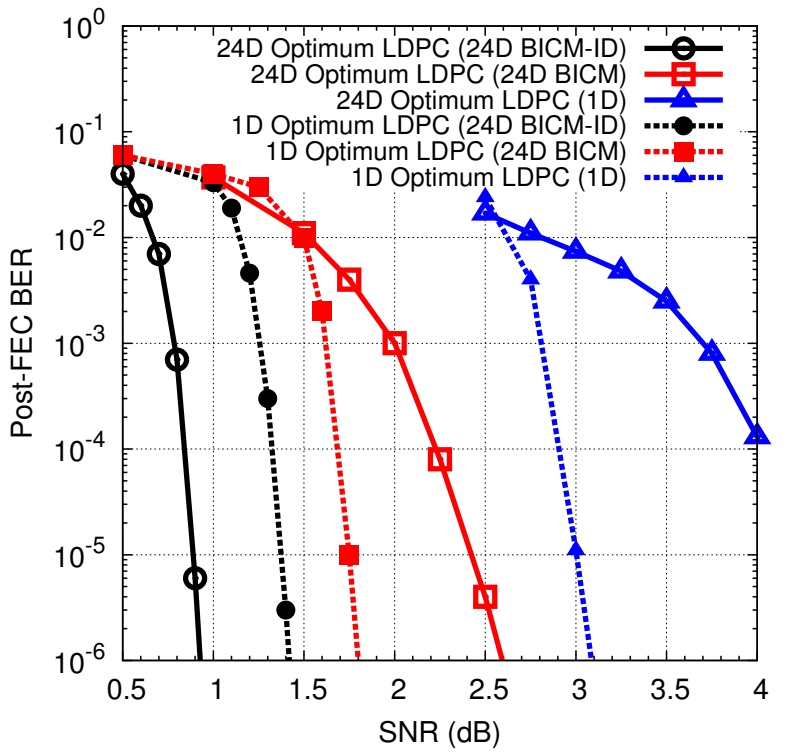

(b) BER vs. SNR

Fig. 2: Modulation-dependent LDPC design for BICM and BICM-ID with high-dimensional modulation.

with the 24D modulation. Here, we also plot the performance of 1D modulation, i.e., binary PSK. Our 24D-optimized code with 24D modulation achieves 2dB gain for BICM-ID, compared to 1D-optimized code with 1D modulation. Moreover, it is shown that the 1D-optimized code may suffer from $0.5 \mathrm{~dB}$ loss from the 24D-optimized code for the case of BICM-ID, while the 24D-optimized code becomes much worse than 1D-optimized code for the case of BICM. The noteworthy point here is that we should use carefully-designed LDPC code depending on modulation schemes (e.g. 1D or 24D) and decoding schemes (e.g., BICM or BICM-ID), in order to achieve the best-possible performance.

\section{Conclusions}

We have shown a significant benefit of designing iteration-dependent and modulation-dependent LDPC codes. We have analyzed thresholds by EXIT chart for BICM and BICM-ID with finite-iteration BP decoders and different HDMs. It has been demonstrated that if we keep using one LDPC code optimized at a certain condition, we can suffer from a large penalty close to $2 \mathrm{~dB}$ loss when the condition such as decoding methods changed. To exploit full potentials of HDM and BP decoding, we may need to carefully design coded modulations depending on situations.

\section{References}

1. I.B. Djordjevic, $O F C$ W3J-4 (2014)

2. S.-Y. Chung, G.D. Forney Jr, T.J. Richardson, R. Urbanke, IEEE COMLET 52 (2001)

3. T.J. Richardson, M.A. Shokrollahi, R.L. Urbanke, IEEE TIT 47 2, (2001)

4. S. ten Brink, G. Kramer, A. Ashikhmin, IEEE TCOMM 524 (2004)

5. L. Schmalen, V. Aref, J. Cho, K. Mahdaviani, ECOC Th.1.3.3 (2014)

6. K. Sugihara, Y. Miyata, T. Sugihara, K. Kubo, H. Yoshida, W. Matsumoto, T. Mizuochi, OFC, OM2B.4 (2013)

7. E. Agrell, M. Karlsson, IEEE JLT 27, 5115-5126 (2009)

8. L. Beygi, E. Agrell, J.M. Kahn, M. Karlsson, IEEE JLT 32 2, 333-343 (2013)

9. T. Liu, I.B. Djordjevic, IEEE Photon. J. 64 (2014)

10. D.S. Millar, T. Koike-Akino, S.Ö Arık, K. Kojima, K. Parsons, T. Yoshida, T. Sugihara, Opt. Exp. 227 (2014)

11. T. Koike-Akino, V. Tarokh, IEEE ICC (2009)

12. A.D. Shiner et al., Opt. Exp. 2217 (2014)

13. C. Dorize, P. Layec, G. Charlet, ECOC Mo.3.5.3 (2014)

14. H. Xiao, A.H. Banihashemi, IEEE COMLET 8 12, 715-717 (2004) 